\title{
Calidad de vida en pacientes con aneurismas intracraneales: cirugía versus tratamiento endovascular
}

\author{
M.J. Katati; S. Santiago-Ramajo*; E. Saura; A. Jorques; M. Pérez-García*; J.M. Martín-Linares; A. Mínguez- \\ Castellano; F. Escamilla-Sevilla y V. Arjona \\ Hospital Virgen de las Nieves. Granada. *Departamento de Personalidad, Evaluación y Tratamiento. Facultad de Psicología de la Universidad \\ de Granada.
}

\section{Resumen}

Objetivo. Basados en estudios anteriores, nuestro propósito es determinar la calidad de vida de los pacientes con aneurismas intracraneales después de haber sido intervenido mediante cirugía o embolización y comparar la calidad de vida de estos dos grupos.

Material y métodos. El cuestionario SF-36 fue aplicado retrospectivamente a 93 pacientes con aneurismas tratados mediante cirugía $(n=56)$ o embolización $(\mathrm{n}=37)$.

Resultados. La calidad de vida de los pacientes tratados con cirugía estaba deteriorada en el $50 \%$ y en los pacientes tratados por embolización el $40,5 \%$ no mostraron ningún deterioro en ninguno de los dominios del SF-36. Los análisis muestran que el dominio de la función física está menos deteriorada en los pacientes que han sido embolizados que los que han sido intervenidos quirúrgicamente.

Conclusiones. Los pacientes con aneurismas intracraneales tratados mediante cirugía o embolización tienen afectada la calidad de vida. El tratamiento endovascular puede causar menos limitaciones en la función física. En la calidad de vida de estos pacientes influyen varios factores, además del tipo de tratamiento.

PALABRAS CLAVE: Calidad de vida. Aneurismas intracraneales. Hemorragia subaracnoidea. Cirugía. Endovascular

Quality of life in intracranial aneurysm: surgery versus endovascular treatment

\section{Summary}

Background. Based on earlier studies, we aimed to determine the quality of life of patients with intracranial aneurysm after their treatment by surgery or

Recibido; 18-10-05. Aceptado: 12-12-05 embolization and to compare the quality of life of these two groups.

Methods. The SF-36 health questionnaire was retrospectively applied to 93 patients with intracranial aneurysm treated with surgery $(n=56)$ or embolization $(\mathrm{n}=37)$.

Results. The quality of life of some patients was impaired but $50 \%$ of patients treated with surgery and $40,5 \%$ of patients treated with embolization showed no impairment in any SF-36 domain. The quality of life in the Physical Functioning domain was higher in embolization-treated than in surgery-treated patients.

Conclusions. Some patients with intracranial aneurysms treated with surgery or endovascular embolization have an impaired quality of life. Endovascular treatment may cause less limitation in physical function. The quality of life of these patients is affected by numerous factors, in addition to the type of treatment.

KEY WORDS: Quality of life. Intracranial aneurysm. Subarachnoid haemorrhage. Surgery. Endovascular. Embolization.

\section{Introducción}

El concepto de calidad de vida ha sido profundamente estudiado desde diferentes disciplinas en los últimos 30 años $^{25}$. En la medicina tradicional solo se consideraban los resultados de una intervención mediante los conceptos de curación, remisión y recurrencia, entre otros ${ }^{7}$. Esta visión de los resultados de una intervención no tiene en cuenta la repercusión que ha tenido la enfermedad en la vida del paciente en diversas áreas como son la familiar, social o laboral. La calidad de vida relacionada con la salud (CVRS) se ha definido como "el valor asignado a la duración de la vida modificado por la deficiencia, el estado funcional, la percepción de salud y la oportunidad social, debido a una enfermedad, accidente o tratamiento determinado"11, y está comprendida por cuatro dimensiones fundamentales que abarcan distintas áreas importantes de la vida del paciente como son el área física, funcional, psicológica y social, que 
dependen de la percepción subjetiva de cada individuo. La investigación en calidad de vida es útil para comprender las reacciones del paciente ante la enfermedad y también para evaluar la eficacia de las intervenciones terapéuticas ${ }^{11}$.

La hemorragia subaracnoidea causada por un aneurisma cerebral es una importante causa de muerte, y ocurre con mayor frecuencia entre los 40 y 60 años de edad. $\mathrm{Su}$ incidencia anual se establece entre 10 y 11 por cada 100.000 habitantes $^{6}$. Es conocido que los pacientes que han sufrido hemorragia subaracnoidea y han sido tratados quirúrgicamente pueden presentar déficit neurológicos, falta de iniciativa, fatiga, irritabilidad, cambio de personalidad o alteraciones emocionales, entre otras ${ }^{10,18,19}$, que afectan negativamente a su calidad de vida. En los últimos 25 años, los avances en el tratamiento de los aneurismas cerebrales ha reducido su mortalidad y morbilidad ${ }^{19}$. Con la introducción del tratamiento endovascular en $1991^{13}$, se ha dirigido el interés de la investigación no sólo a la morbilidad-mortalidad de este procedimiento, sino también a su impacto en la calidad de vida de los pacientes y su comparación con la cirugía. Un estudio pionero en comparar las dos técnicas es el Internacional Subarachnoid Aneurysm Trial (ISAT) ${ }^{20}$, donde llegan a la conclusión que las consecuencias negativas, de dependencia y muerte, son mayores después del tratamiento quirúrgico comparado con el tratamiento endovascular. Desde la publicación del ISAT, han aparecido numerosos trabajos que no están totalmente de acuerdo con la metodología y los resultados obtenidos ${ }^{22,23,28,29}$ asegurando que no es suficiente con los resultados del ISAT para proclamar la superioridad del tratamiento endovascular.

Este trabajo pretende esclarecer si existen diferencias en la calidad de vida atribuibles a la modalidad de tratamiento (cirugía versus embolización) en pacientes con aneurismas cerebrales medido con un cuestionario muy utilizado en este ámbito (SF-36), que mide distintos ámbitos importantes de la vida del paciente.

\section{Material y métodos}

\section{Pacientes}

Entre 1995 y Febrero de 2002 se trataron 204 pacientes con aneurismas cerebrales en el servicio de Neurocirugía del Hospital Universitario Virgen de las Nieves. Cien pacientes aceptaron participar en este estudio retrospectivo, y finalmente se incluyen para este trabajo 93 (45,59\%), ya que 2 pacientes presentaban otras alteraciones que tenían asociados déficits neuropsicológicos (Parkinson y traumatismo craneoencefálico), 1 no hablaba correctamente la lengua española, 1 presentaba un síndrome afásico que impidió la aplicación de toda la batería y 3 mostraron falta de colaboración durante la evaluación. Los pacientes recibieron tratamiento quirúrgico o endovascular según su adecua- ción. 56 de los pacientes fueron tratados quirúrgicamente $(60,22 \%)$, mientras que los restantes 37 fueron tratados con embolización (39,78\%). Las características demográficas, clínicas y quirúrgicas de los pacientes se muestran en las tablas 1 y 2 .

\section{Material}

\section{SF-36}

El Cuestionario de Salud SF-36 fue desarrollado por Medical Outcome Study (MOS) por Ware y Sherbourne ${ }^{32}$. La adaptación a la población española siguió el protocolo común a todos los países que participaron en el International Quality of Life Assessment -IQOLA-Projetct ${ }^{1,31}, \mathrm{y}$ que se encuentra publicada por Alonso y colaboradores en $1995^{2}$.

El SF-36 contiene 36 ítems formando 8 dimensiones que evalúan:

- Función física: grado en que la salud limita las actividades físicas tales como el autocuidado, caminar, subir escaleras, inclinarse, coger o llevar pesos y los esfuerzos moderados e intensos (10 ítems).

- Rol físico: grado en que la salud física interfiere en el trabajo y otras actividades diarias, incluyendo rendimiento menor que el deseado, limitación en el tipo de actividades realizadas o dificultad en la realización de actividades (4 ítems).

- Dolor corporal: intensidad del dolor y su efecto en el trabajo habitual, tanto fuera de casa como en el hogar (2 ítems).

- Salud general: valoración personal de la salud que incluye la salud actual, las perspectivas de salud en el futuro y la resistencia a enfermar (5 ítems).

- Vitalidad: sentimiento de energía y vitalidad, frente al sentimiento de cansancio y agotamiento (4 ítems).

- Función social: grado en que los problemas de salud física o emocional interfieren en la vida social habitual (2 ítems).

- Rol emocional: grado en que los problemas emocionales interfieren en el trabajo u otras actividades diarias, que incluye la reducción en el tiempo dedicado a esas actividades, el rendimiento menor que el deseado y una disminución del cuidado al trabajar. (3 ítems).

- Salud mental: salud mental general, que incluye depresión, ansiedad, control de la conducta y el efecto positivo en general (5 ítems).

No se ha tenido en cuenta el ítem de la evolución de la enfermedad (1 ítem) por no constituir una dimensión aparte.

\section{Procedimiento}

Procedimiento quirúrgico y endovascular

En la unidad de cirugía vascular de nuestro hospital, 
Tabla 1

Diferencias en la distribución de las variables demográficas y clínicas entre los grupos de tratamiento quirúrgico y endovascular.

\begin{tabular}{|c|c|c|c|}
\hline Variable & Cirugía $n(\%)$ & Embolización $n(\%)$ & $p$ \\
\hline \multicolumn{4}{|l|}{ Sexo } \\
\hline Mujer & $34(60,7)$ & $19(51,4)$ & \multirow[t]{2}{*}{ ns } \\
\hline Hombre & $22(39,3)$ & $18(48,6)$ & \\
\hline \multicolumn{4}{|l|}{ Dominancia Manual } \\
\hline Diestro & $52(92,9)$ & $37(100)$ & \multirow[t]{2}{*}{$<, 150$} \\
\hline Zurdo & $4(7,1)$ & $0(0)$ & \\
\hline \multicolumn{4}{|l|}{ Localización del aneurisma } \\
\hline Comunicante anterior & $30(53,6)$ & $10(27)$ & \multirow{5}{*}{, 013} \\
\hline Cerebral media & $11(19,6)$ & $5(13,5)$ & \\
\hline Comunicante posterior & $4(7,1)$ & $10(27)$ & \\
\hline Bifurcación carótida & $4(7,1)$ & $2(5,4)$ & \\
\hline Otras & $7(12,5)$ & $10(27)$ & \\
\hline \multicolumn{4}{|l|}{ Hunt \& Hess } \\
\hline 0 (intacto) & $5(8,9)$ & $4(10,8)$ & \multirow[t]{3}{*}{ ns } \\
\hline 1-3 (moderado) & $42(75)$ & $27(73)$ & \\
\hline 4-5 (severo) & $9(16,1)$ & $6(16,2)$ & \\
\hline \multicolumn{4}{|l|}{ Fisher } \\
\hline 1 & $11(19,6)$ & $6(16,2)$ & \multirow{4}{*}{$<, 150$} \\
\hline 2 & $13(23,2)$ & $12(32,4)$ & \\
\hline 3 & $17(30,49)$ & $4(10,8)$ & \\
\hline 4 & $15(26,8)$ & $15(40,5)$ & \\
\hline \multicolumn{4}{|l|}{ Miscelaneas/Complicaciones } \\
\hline Drenaje ventricular & $4(7,1)$ & $5(13,5)$ & ns \\
\hline DVP & $11(19,6)$ & $7(18,9)$ & ns \\
\hline Resangrado antes tratamiento & $1(1,8)$ & $1(2,7)$ & ns \\
\hline Hidrocefalia & $11(19,6)$ & $7(18,9)$ & ns \\
\hline Vasoespasmo & $7(12,5)$ & $13(35,1)$ &, 014 \\
\hline Infarto & $7(12,5)$ & $8(21,6)$ & $\mathrm{ns}$ \\
\hline Rotura intraoperatoria & $2(3,6)$ & $0(0)$ & ns \\
\hline \multicolumn{4}{|l|}{ GOS al alta } \\
\hline 3 & $5(8,9)$ & $4(10,8)$ & \multirow{3}{*}{ ns } \\
\hline 4 & $24(42,9)$ & $11(29,7)$ & \\
\hline 5 & $27(48,2)$ & $22(59,5)$ & \\
\hline \multicolumn{4}{|l|}{ GOS en la evaluación } \\
\hline 2 & $0(0)$ & $1(2,7)$ & \multirow{4}{*}{ ns } \\
\hline 3 & $0(0)$ & $1(2,7)$ & \\
\hline 4 & $6(10,7)$ & $6(16,2)$ & \\
\hline 5 & $50(89,3)$ & $29(78,4)$ & \\
\hline
\end{tabular}

ns = no significativo; DVP = drenaje ventrículoperitoneal 
Tabla 2

Diferencias en las medias de las variables clínicas y demográficas de los grupos de tratamiento quirúrgico y endovascular

\begin{tabular}{llcccc}
\hline Variable & Media & DS & Min - Max & $p$ \\
\hline Edad en la evaluación & & & & & \\
$\quad$ Cirugía & 54 & 11,10 & 28 & 78 & $\mathrm{~ns}$ \\
$\quad$ Embolización & 54 & 13,64 & 26 & 78 & \\
\hline Edad en el tratamiento & & & & & \\
$\quad$ Cirugía & 49,55 & 11,55 & 24 & 75 & $\mathrm{~ns}$ \\
$\quad$ Embolización & 50,43 & 13,71 & 20 & 75 & \\
\hline Tiempo de evolución (años) & & & & & $\mathrm{ns}$ \\
$\quad$ Cirugía & 3,95 & 2,23 & 1 & 7,92 & \\
$\quad$ Embolización & 3,69 & 1,44 & 1,7 & 6,17 & $\mathrm{n}$ \\
Años de escolaridad & & & & & \\
$\quad$ Cirugía & 6,75 & 4,49 & 0 & 16 & \\
$\quad$ Embolización & 8 & 5,24 & 0 & 18 & \\
\hline
\end{tabular}

DS = Desviación estándar; Min — Man = Mínimo y Máximo; ns = no significativo

inicialmente todos los pacientes con aneurismas cerebrales son valorados para tratamiento endovascular. El tratamiento quirúrgico se realiza en los casos en los que la embolización no puede llevarse a cabo por ser el aneurisma de cuello ancho o por la presencia de algún vaso eferente del propio saco aneurismático. Durante el periodo indicado (1995-2002) no se ha producido modificación en la técnica ni la metodología en los dos procedimientos citados.

\section{Evaluación de la calidad de vida}

Tras haber transcurrido un mínimo de un año después del tratamiento se citó a los pacientes para realizar una evaluación de la calidad de vida. El SF-36 se administró en forma de entrevista por un neuropsicólogo entrenado.

Las puntuaciones directas de cada paciente se convirtieron a puntuaciones estandarizadas llamadas puntuaciones $\mathrm{Z}$ $($ Media $=0$, Desviación Típica $=1)$, que permiten conocer la ejecución del paciente corrigiendo el efecto de la edad y el sexo. Para esta conversión se emplearon los datos normativos publicados para población española ${ }^{3}$.

\section{Análisis estadísticos}

En primer lugar, se hallarán las frecuencias y porcentajes de los sujetos con deterioro en los dominios del SF-36 en los grupos de tratamiento quirúrgico y endovascular. Se considerará que el dominio está deteriorado si la puntuación está 2 SD por debajo de la media $(Z<-2)$ de acuerdo con los criterios del estudio de normalización3.

En segundo lugar, con el objetivo de establecer si ambos grupos son homogéneos en los diferentes factores demográficos, clínicos y quirúrgicos, se realizarán análisis de contingencia para variables nominales y t de Student para variables continuas.

En tercer lugar, para estudiar si existían diferencias en calidad de vida entre los tratamientos, se realizaron diversos análisis. El primer paso fue realizar ocho análisis "Set-Wise" mediante el programa Minitab 14, uno por cada dominio del SF-36. Por lo tanto, para cada análisis se tomó como variable dependiente un dominio del SF-36 y como predictores se seleccionaron aquellas variables clínicas o demográficas en las que había diferencias estadísticamente significativas entre el grupo quirúrgico y el endovascular. Además, para estudiar la posible influencia de otras variables que estaban relacionadas aunque no eran diferentes entre los grupos, se decidió incluir como predictor aquellas variables cuya $p$ fuera menor de, 150 . De los resultados de este análisis se seleccionó, para cada dominio, el modelo con una R-Sq (adj) más alta. En aquellos dominios en que la R-Sq (adj) era ,00 para todas las variables, no se escogió ningún modelo $\mathrm{y}$, por tanto, no se siguió realizando ningún análisis.

El segundo paso consistió en comprobar si el modelo seleccionado por cada uno de los dominios del SF-36, predecía la variable dependiente usando regresiones jerárquicas múltiples. Como variable dependiente se utilizaron los distintos dominios del SF-36 y como variables independientes o predictoras el modelo seleccionado del procedimiento "Set-Wise".

Por último, para todos los análisis se ha realizado el ajuste de Bonferroni, quedando fijado el nivel de significación en ,006. 


\section{Resultados}

En primer lugar, con el objetivo de describir la calidad de vida de los pacientes, se determinó el número de pacientes que presentan deteriorada la calidad de vida en alguno de los dominios de la SF-36. En la tabla 3 se muestra el número de pacientes que presentan $2 \mathrm{SD}$ inferiores a la media (puntuación $Z<-2$ ). Para cada dominio se ha utilizado los datos normativos publicados para la población española ${ }^{3}$. Como se observa, los dominios más frecuentemente afectados en los pacientes tratados quirúrgicamente son la Función Social $(32,1 \%)$ y el Rol Emocional $(30,4 \%)$. En cambio, los dominios con menor frecuencia de deterioro son la Función Física $(7,1 \%)$ y la Salud General $(7,1 \%)$. En el grupo de pacientes tratados endovascularmente los dominios con mayor frecuencia de deterioro son de nuevo los dominios Función Social $(32,4 \%)$ y Rol Emocional $(32,4 \%)$. Los dominios menos afectados son la Función Física $(0 \%)$ y la Vitalidad $(5,4 \%)$.

En segundo lugar, con el objetivo de determinar si los grupos eran homogéneos en características clínicas y demográficas, se realizaron 13 análisis de contingencia y 4 t de Student. Dichos análisis no resultaron estadísticamente significativos, excepto en la distribución de la localización del aneurisma $\left(\chi^{2}=12,664, \mathrm{p}=, 013\right)$ y el vasoespasmo $(\chi 2=6,004, p=, 014)$. No obstante las variables Dominan-
Tabla 3

Frecuencia y porcentajes de deterioro en los dominios del SF-36 en los grupos de tratamiento quirúrgico y endovascular

\begin{tabular}{lcc}
\hline Variable & $\begin{array}{c}\text { Cirugía } \\
n(\%)\end{array}$ & $\begin{array}{c}\text { Embolización } \\
n(\%)\end{array}$ \\
\hline Función Física & $4(7,1)$ & $0(0)$ \\
Rol Físico & $11(19,6)$ & $10(27)$ \\
Dolor Corporal & $12(21,4)$ & $7(18,9)$ \\
Salud General & $4(7,1)$ & $3(8,1)$ \\
Vitalidad & $7(12,5)$ & $2(5,4)$ \\
Función Social & $18(32,1)$ & $12(32,4)$ \\
Rol Emocional & $17(30,4)$ & $12(32,4)$ \\
Salud Mental & $7(12,5)$ & $3(8,1)$ \\
\hline
\end{tabular}

cia manual y la extensión de la hemorragia subaracnoidea indicada por la escala de Fisher, tienen un $\mathrm{p}<, 150 \mathrm{y}$, por lo tanto, serán incluidas en los análisis posteriores por las razones que ya se han explicado en el apartado de Análisis estadísticos. En las tablas 1 y 2 se resumen las características principales de cada uno de los dos grupos de tratamiento, y si existen diferencias estadísticamente significativas en cada variable entre ambos grupos.

Tabla 4

Procedimiento "Set-Wise" y regresiones jerárquicas múltiples para los ocho dominios del SF-36

\begin{tabular}{|c|c|c|c|c|c|c|c|c|c|c|c|}
\hline \multirow{2}{*}{ VARIABLE } & \multicolumn{5}{|c|}{ PREDICTORES } & \multirow{2}{*}{$\mathrm{R}-\mathrm{Sq}$} & \multirow{2}{*}{ C- $p$} & \multicolumn{2}{|c|}{ MODELO } & \multicolumn{2}{|c|}{ CAMBIO } \\
\hline & 1 & 2 & 3 & 4 & 5 & & & $\mathrm{~F}$ & $p$ & $\mathrm{~F}$ & $p$ \\
\hline \multirow[t]{2}{*}{ Función Física } & & & $\mathrm{X}$ & & & & & 7,283 &, 008 & 7,283 & ,008 \\
\hline & $\mathrm{X}$ & & $\mathrm{X}$ & & & 11,4 & 2,2 & 6,958 &, $002 *$ & 6,215 &, 014 \\
\hline \multirow[t]{2}{*}{ Rol Físico } & & & & & $\mathrm{X}$ & & & 2,680 & , 105 & 2,680 & , 105 \\
\hline & & & & $X$ & $\mathrm{X}$ & 2,5 &, 8 & 2,197 &, 117 & 1,694 & , 196 \\
\hline Dolor Corporal & & & & & & 0 & & & & & \\
\hline Salud General & & & & & & 0 & & & & & \\
\hline \multirow[t]{2}{*}{ Vitalidad } & & $\mathrm{X}$ & & & & & & 1,704 & , 195 & 1,704 & , 195 \\
\hline & & $\mathrm{X}$ & $\mathrm{X}$ & & & 2,8 & 1,8 & 2,344 &, 102 & 2,947 & ,089 \\
\hline Función Social & & $\mathrm{X}$ & & & & 1,0 &,- 8 & 1,881 &, 174 & 1,881 & , 174 \\
\hline Rol Emocional & & & & & & 0 & & & & & \\
\hline \multirow[t]{2}{*}{ Salud Mental } & & & & & $\mathrm{X}$ & & & ,714 & ,399 & ,717 & ,399 \\
\hline & $\mathrm{X}$ & & & & $\mathrm{X}$ & 5,4 & 1,6 & ,386 & 681 &, 062 & ,803 \\
\hline
\end{tabular}

Predictores

1 Tipo de intervención

2 Dominancia manual

3 Escala Fisher

4 Localización del aneurisma

5 Vasoespasmo

* estadísticamente significativo por el ajuste de Bonferroni $\mathrm{p}<, 006$ 
En tercer lugar, para determinar la existencia de diferencias en la calidad de vida debidas a la modalidad de tratamiento recibido (endovascular versus quirúrgico) se realizaron diversos análisis. Primero, se aplicó el procedimiento de "set-wise" para obtener el modelo más predictivo para cada dominio de la calidad de vida (SF-36). Por ello, se incluyeron como predictores las dos variables que son estadísticamente diferentes entre los dos grupos (localización del aneurisma y presencia de vasoespasmo), las dos variables que tienen una $\mathrm{p}<, 150$ (dominancia manual y escala Fisher) y la variable de interés para nuestro estudio (tipo de tratamiento). El procedimiento se realizó con cada uno de los ocho dominios del SF-36 y con las cinco variables comentadas. Los resultados se muestran en la tabla 4.

Por último, se estudió el modelo seleccionado para ver si predecía significativamente la variable dependiente utilizando una regresión jerárquica múltiple. Los resultados muestran que el único modelo que resultó significativo fue la variable dependiente función física con dos variables predictoras: la escala de Fisher y el tipo de intervención (endovascular versus quirúrgico) $(\mathrm{F}(2,90)=6,958 ; \mathrm{p}<, 002)$. En la regresión jerárquica múltiple de este modelo se incluyeron dos bloques: el primer bloque incluye la escala Fisher y el segundo bloque la escala Fisher y el tipo de tratamiento. El resultado para el segundo bloque introducido mostró que la variable modalidad de tratamiento resulta predictora a pesar del efecto de la severidad de la hemorragia $(p<, 014)$. Los demás dominios no resultaron significativos con ninguna de las variables predictoras introducidas.

\section{Discusión}

El objetivo de la investigación fue determinar la existencia de diferencias en la calidad de vida en función de la modalidad de tratamiento que recibieran los pacientes (cirugía versus embolización). El grupo de tratamiento endovascular presenta menos limitaciones en la función física que el grupo de tratamiento quirúrgico. De hecho, ninguno de los pacientes que recibieron tratamiento endovascular mostró daño en esta variable, que mide el grado en que la salud limita las actividades físicas tales como el autocuidado, caminar, subir escaleras, inclinarse, coger o llevar pesos y los esfuerzos moderados e intensos. Los dominios más afectados son la función social y el rol emocional, que aparecen deteriorados en más del 30\% de los pacientes. Nuestros datos coinciden con los aportados por otros trabajos, que indican la presencia de alteraciones sociales y emocionales en estos pacientes ${ }^{12,14,16,17,26}$. Concretamente, en el estudio de Hop y cols. $(2001)^{16}$, los resultados del estudio realizado mostraron que las puntuaciones eran significativamente más bajas en los dominios de función social, limitación del rol físico y limitación del rol emocional, en el grupo de pacientes que habían sufrido una hemorragia subaracnoidea después de 18 meses. Resultados similares se mostraron en el estudio de Hackkett y Anderson (2000) ${ }^{14}$, donde el grupo de pacientes con hemorragia subaracnoidea mostraron puntuaciones más bajas en limitación del rol físico y limitación del rol emocional medido después de un año.

Hay que tener en cuenta que, aunque en nuestro estudio ambos grupos son muy similares, los análisis previos mostraron que diferían significativamente en la localización del aneurisma y en la presencia de vasoespasmo. Los resultados mostraron que la modalidad de tratamiento solo resulta predictiva en el dominio función física. De este modo, el grupo de tratamiento quirúrgico presenta menores puntuaciones que el grupo de tratamiento endovascular. El único trabajo que conocemos que haya abordado las diferencias entre ambos tratamientos en calidad de vida con pacientes que han sufrido una hemorragia subaracnoidea, encontró en los pacientes tratados endovascularmente una tendencia no significativa a presentar mejor calidad de vida ${ }^{21}$. No obstante, esos resultados son difícilmente comparables con el presente trabajo dado que los instrumentos de evaluación son muy diferentes y aportan información no comparable. Otro trabajo similar ${ }^{5}$, pero con pacientes con aneurismas no rotos, llega a la conclusión que los pacientes intervenidos quirúrgicamente tiene afectada negativamente su calidad de vida, en cambio, los que fueron embolizados no vieron afectados su calidad de vida.

Por lo tanto, encontramos que la modalidad de tratamiento apenas afecta a la calidad de vida de los pacientes exceptuando el dominio función física. Este efecto podría explicarse como consecuencia de la oclusión temporal de los vasos sanguíneos, como indica Hütter y cols. $(2001)^{18}$ que, tras estudiar la calidad de vida de pacientes intervenidos quirúrgicamente, sugieren que el tiempo de la oclusión temporal durante la cirugía se relaciona con un mayor riesgo de afectación permanente de la calidad de vida.

No obstante, el modelo predictivo empleado en nuestro estudio explica un porcentaje de la varianza muy bajo, por lo que creemos que existen otros factores no asociados a la modalidad de intervención que determinan el deterioro de la calidad de vida de estos pacientes. El grado de afectación de la calidad de vida parece asociarse al tiempo transcurrido desde la intervención. El 50\% de los pacientes muestran una mejoría en la calidad de vida relacionada con una mejora en la escala de Rankin a los 18 meses $^{18}$. También se ha relacionado una peor calidad de vida con factores clínicos como el estado neurológico al ingreso medido con la escala Hunt \& Hess, la intensidad de la hemorragia inicial, la severidad de la hemorragia (Fisher), la edad, discapacidad física (Rankin), daño cognitivo en general o con estados de ánimo depresivos ${ }^{8,15,18,19,24,26}$. En un estudio más reciente, Schuiling y cols. $(2005)^{30}$ encontraron que la peor calidad de vida de estos pacientes se veía afectada 
por los trastornos del sueño. Pero a pesar de esto, hay un estudio ${ }^{9}$ que afirma la existencia de correlaciones de dos factores con la calidad de vida de estos pacientes, que son el GOS y el déficit neurológico pero no encuentra relación con la escala de Hunt\&Hess y la escala de Fisher. Además, hay que tener en cuenta el trastorno de estrés postraumático causado por la propia percepción de enfermedad después de sufrir la hemorragia subaracnoidea que afecta también negativamente a la calidad de vida, según Berry $(1998)^{4}$ y Pritchard y cols. (2004) ${ }^{27}$. Lo que podemos concluir de todos estos estudios, es que la calidad de vida en estos pacientes no sólo depende de un factor, sino que interfieren muchos factores de diverso tipo, que todos afectan en diferente medida a la calidad de vida deteriorada de los pacientes que han sido tratados de un aneurisma intracraneal y que la modalidad de tratamiento solo explica una parte.

Por último, queremos resaltar que los diferentes hallazgos han de ser considerados con cautela debido a las limitaciones que presenta este trabajo. En primer lugar es un estudio retrospectivo, por lo tanto, el tiempo transcurrido desde la intervención hasta la medición de la calidad de vida puede implicar a otros factores que puedan estar afectando a la calidad de vida. Lo que demuestra la necesidad de estudios prospectivos en esta área que intenten controlar todos los factores que puedan intervenir en la calidad de vida. Además, se ha perdido la información de numerosos pacientes que no pudieron participar. Es posible que algunos de los pacientes que no participaran en este trabajo no lo hicieran por encontrarse más limitados al padecer secuelas más graves o, al contrario, porque recuperaran totalmente su nivel de vida anterior y no consideraran necesario participar en el estudio. Por lo tanto, no podemos asegurar que estos datos sean aplicables a toda la población de pacientes. Metodológicamente hemos de considerar que hubiera sido adecuado asignar aleatoriamente los pacientes a las modalidades de tratamiento quirúrgico o endovascular. En cambio, este procedimiento hubiera llevado a la exclusión del estudio de aquellos pacientes que no podían ser distribuidos de forma aleatoria, ya que por las características del aneurisma se hacía necesaria una sola modalidad de tratamiento. Así, en nuestro trabajo, si bien la adecuación metodológica es menor, se describe un rango más amplio de población con hemorragia subaracnoidea, al incluirse aquellos pacientes que necesariamente habían de recibir tratamiento quirúrgico. Además, para controlar las variables en las que ambos grupos no eran homogéneos se han aplicado procedimientos estadísticos para controlar el efecto de las variables clínicas y demográficas en las que diferían los grupos.

En conclusión, hemos encontrado que. en nuestra serie de pacientes, la calidad de vida se ve deteriorada. La modalidad de tratamiento parece afectar a la calidad de vida de forma estadísticamente significativa en la dimensión física, que se muestra más deteriorada en el grupo tratado quirúrgicamente. No obstante, el porcentaje de varianza explicado por la modalidad de tratamiento es muy bajo, y por lo tanto, consideramos que hay implicadas además otras variables que determinan la calidad de vida de estos pacientes.

\section{Bibliografía}

1. Aaronson, N.K., Acquadro, C., Alonso, J., et al : International quality of life assessment (IQOLA) project. Qual Life Res 1992; 1: 349-351.

2. Alonso, J., Prieto, L., Antó, J.M.: La versión española del SF-36 Health Survey (Cuestionario de Salud SF-36): un instrumento para la medida de los resultados clínicos. Med Clin (Bart) 1995; 104: 771-776.

3. Alonso, J., Regidor, E., Barrio, G., Prieto, L., Rodríguez, C., de la Fuente, L.: Valores poblacionales de referencia de la versión española del Cuestionario SF-36. Med Clin (Bart) 1998; 111: 410-416.

4. Berry, E.: Post-traumatic stress disorder after subarachnoid haemorrhage. Br J Clin Psychol 1998; 37: 365-367.

5. Brilstra, E.H., Rinkel, G.J., Van der Graaf, Y. et al.: Quality of life after treatment of unruptured intracranial aneurysms by neurosurgical clipping or by embolisation with coils. Cerebrovasc Dis 2004; 17: 44-52.

6. Cardentey-Pereda, A. L., Pérez-Falero, R. A.: Hemorragia subaracnoidea. Rev Neurol 2002; 34: 954-966.

7. Carod-Artal, F.J.: Medición de la calidad de vida en supervivientes de un ictus. Rev Neurol 1999; 29: 447-456.

8. Carter, B.S., Buckley, D., Ferraro, R., Rordorf, G., Ogilvy, C.S.: Factors associated with reintegration to normal living alter subarachnoid hemorrhage. Neurosurgery 2000; 46: 1326-1334.

9. Cedzich, C., Roth, A.: Neurological and psichosocial outcome after subarachnoid haemorrhage, and the hunt and hess scale as a predictor of clinical outcome. Zentralbl Neurochir 2005; 66: 112-118.

10. DeLuca, J.: Cognitive dysfunction after aneurysm of the anterior communicating artery. J Clin Exp Neuropsychol 1992; 14 : 924-934.

11. Fernández-Concepción, O., Fiallo-Sánchez, M.C., Alvarez-González, M.A., Roca, M.A, Concepción-Rojas, M., Chávez, L.: La calidad de vida del paciente con accidente cerebrovascular: una visión desde sus posibles factores determinantes. Rev Neurol 2001; 32: 725-731.

12. Fertl, E., Killer, M., Eder, H., Linzmayer, L., Richling, B., Auff, E.: Long-term functional effects of aneurysmal subarachnoid haemorrhage with special emphasis on the patient's view. Acta Neurochir (Wien) 1999; 141: 571-577

13. Guglielmi, G., Viñuela, F., Sepetka, I., Macellari, V.: Electrothrombosis of Saccular aneurysms via endovascular approach. J Neurosurg 1991; 75: 1-7. 
14. Hackkett, M.L., Anderson, C.S.: Health Outcome 1 year after subarachnoid hemorrhage: An international population-based study. The Australian Cooperative Research on Hemorrhage Study Group. Neurology 2000; 55: 658-662.

15. Hop, J.W., Rinkel, G.J., Algra, A., van Gijn, J.: Quality of life in patients and partners after aneurysmal subarachnoid hemorrhage. Stroke 1998; 29: 798-804.

16. Hop, J.W., Rinkel, G.J., Algra, A., van Gijn, J.: Changes in functional outcome and quality of life in patients and caregivers after aneurysmal subarachnoid hemorrhage. J Neurosurg 2001; 95: 957-963.

17. Hütter, B.O., Gilsbach, J.M., Kreitschmann, I .: Quality of life and cognitive deficits after subarachnoid haemorrhage. Br J Neurosurg 1995; 9: 465-475.

18. Hütter, B.O., Kreitschmann-Andermahr, I., Gilsbach, J.M.: Health-related quality of life after aneurysmal subarachnoid hemorrhage: impacts of bleeding severity, computerized tomography findings, surgery, vasospasm, and neurological grade. J Neurosurg 2001; 94: 241-251.

19. Hütter, B.O., Kreitschmann-Andermahr, I., Mayfrank, L., Rohde, V., Spetzger, U., Gilsbach, J.M.: Functional outcome after aneurysmal subarachnoid hemorrhage. Acta Neurochir Suppl (Wien) 1999; 72: 157-174.

20. International Subarachnoid Aneurysm Trial (ISAT) Collaborative Group: International Subarachnoid Aneurysm Trial (ISAT) of neurosurgical clipping versus endovascular coiling in 2143 patients with ruptured intracranial aneurysms: a randomised trial. Lancet 2002; 360: 1267-1274.

21. Khára, V.J., Seppanen, S.K., Kuurne, T., Laasonen, E.M.: Patient outcome after endovascular treatment of intracranial aneurysms with reference to microsurgical clipping. Acta Neurol Scand 1999; 99: 284-290.

22. Lindsay, K.W.: The impact of the International Subarachnoid Aneurysm Treatment Trial (ISAT) on neurosurgical practice. Acta Neurochir (Wien) 2003; 145: 97-99.

23. Maurice-Williams, R.S.: Aneurysm surgery after the International Subarachnoid Aneurysm Trial (ISAT). J Neurol Neurosurg Psychiatry 2004; 75: 807-808.

24. Mayer, S.A., Kreiter, K.T., Copeland, D. et al.: Global and domain-specific cognitive impairment and outcome after subarachnoid hemorrhage. Neurology 2002; 59: 1750-1758.

25. Murrell, R.: Quality of life and neurological illness: a review of the literature. Neuropsychol Rev 1999; 9: 209-229.
26. Powell, J., Kitchen, N., Heslin, J., Greenwood, R.: Psychosocial outcomes at three and nine months after good neurological recovery from aneurysmal subarachnoid haemorrhage: predictors and prognosis. J Neurol Neurosurg Psychiatry 2002; 72: 772-781

27. Pritchard, C., Foulkes, L., Lang, D.A, Neil-Dwyer, G.: Two-year prospective study of psychosocial outcomes and a cost-analysis of "treatment-as-usual" versus an "enhanced" (specialist liaison nurse) service for aneurysmal subarachnoid haemorrhage (ASAH) patients and families. B J Neurosurg 2004; 18: 347-356.

28. Raabe, A., Schmiedek, P., Seifert, V., Stolke, D.: German Society of Neurosurgery Section on Vascular Neurosurgery: Position Statement on the International Subarachnoid Hemorrhage Trial (ISAT). Zentralbl Neurochir 2003; 64: 99103.

29. Sade, B., Mohr, G.: Critical appraisal of the International Subarachnoid Aneurysm Trial (ISAT). Neurol India 2004; 52: 32-35.

30. Schuiling, W.J., Rinkel, G.J., Walchenbach, R., de Weerd, A.W.: Disorders of sleep and wake in Patients After Subarachnoid Hemorrhage. Stroke 2005; 36: 578-582.

31. Ware, J.E.Jr., Gandek, B. and the IQOLA Project Group: The SF-36 Health survey: development and use in mental health research and the IQOLA project. Int J Ment Health 1994; 23: 49-73.

32. Ware, J.E.Jr., Sherbourne, C.D.: The MOS 36-item short form health survey (SF-36). Med Care 1992; 30: 473483.

Este trabajo ha sido financiado por la Fundación Virgen de las Nieves (Hospital Virgen de las Nieves)

Katati, M.F.; Santiago-Ramajo,S.; Saura, E.; Jorques, A.; Pérez-García, M.; Martín-Linares, J.M. Mínguez-Castellano, A.; Escamilla-Sevilla, F.; Arjona, V.: Calidad de vida en pacientes con aneurismas intracraneales: cirugía versus tratamiento endovascular. Neurocirugía 2007; 17: $325-332$.

Correspondencia postal: Dr. Majed J. Katati. Servicio de Neurocirugía. Hospital Universitario Virgen de las Nieves Ctra. Jaén s/n. 18012 Granada (España) 